\title{
Fingerprint classification using entropy sensitive tracing
}

\author{
Preda Mihăilescu ${ }^{1}$, Krzysztof Mieloch ${ }^{2}$, and Axel Munk ${ }^{3}$ \\ 1 Mathematical Institute, University of Göttingen, Germany \\ preda@uni-math.gwdg. de \\ 2 Institute for Mathematical Stochastics, University of Göttingen, Germany \\ mieloch@math.uni-goettingen.de \\ 3 Institute for Mathematical Stochastics, University of Göttingen, Germany \\ munk@math . uni-goettingen . de
}

\section{Introduction}

Fingerprints are currently the leading approach to biometric recognition[1]. The reasons are multiple - we mention on the one hand the more than centennial tradition of fingerprint use for forensic purposes and on the other hand the existence of some well established experience - based rules derived along the line. Fingerprints have a specific flow dynamics, which comes in quite distinct flow patterns - these help define classes of fingerprints. The flow pattern carries various singularities, named minutiae - most important are line endings and bifurcations.

This paper treats the classification of fingerprints. In order to establish the identity of a person from a given fingerprint image, it is necessary to search large databases. Hence, the first step is to reduce the search field by assigning a given fingerprint into one of a small number of categories. Then the matching is performed only among fingerprints which belong to the class of the template.

Most of the classification schemas currently used worldwide are variants of Henry's classification scheme[1]. The six most common classes are: tended arch, arch, left loop, right loop, whorl and twin loop.

While finger matching is usually performed with the help of local features, the fingerprints classification is generally based on global features, such as skin ridge flow. For classifications, one may consider orientation field as a feature vector $[2,3,4]$. Different procedures for reduction of the dimension of the orientation field, such as e.g. Karhunen-Loève transformation $[6,7]$ were proposed. With the reduced vector, the statistical classifiers are involved[5, 8]. Further approaches are found in $[9,10],[11]$. 


\section{Tracer}

We developed an approach for fingeprint recognition which begins with the extraction of the essential fingerprint data, organised in semantic structures. We have described the notions and objects for this novel fingerprint data extraction denoted entracing in [12].

The extracted data contains not only minutiae information but also information about interminutiae connections (ridges) as complete connected lines. The flow of these lines is used in this paper for classification.

\section{Characteristic lines}

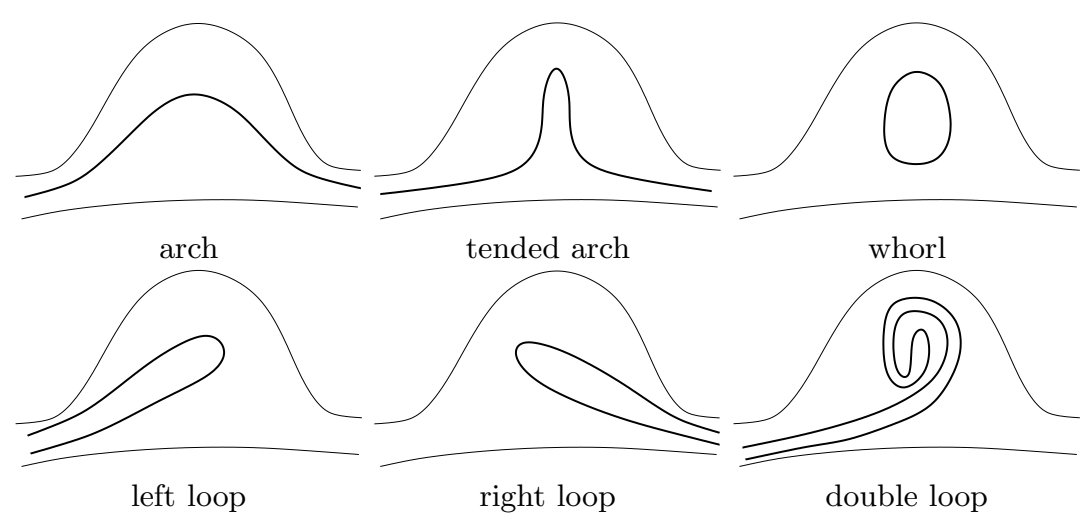

Fig. 1. Classes according to Henry's classification scheme.

An expert analyst's experience suggested that every Henry class has certain windings of connected lines which are specific and do not occur in other classes. They are to be found typically around the centre of the finger (see Fig. 1). This suggests the fact that, using the entraced connected lines, one may have a quick search for class - characteristic lines.

Guided by these insight, the research questions which we begin to address in the present paper are the following:

A. What simple metric characterisation can be used for defining distinctive, class dependent, connected line flows?

B. How accurate is the statistical classification based on the given metric?

C. When applied to large databases, how close can the new method approach the state of the art performances. Or, can one approach the intrinsic limits of classification? ${ }^{4}$

\footnotetext{
${ }^{4}$ It is well known that there are fingerprints which cannot be uniquely assigned to classes, while others cannot be assigned to any class, even by the human expert.
} 


\subsection{Defining characteristic lines}

As a result of tracing we get a usually disconnected graph $G=\{V, E\}$ where each vertex $v \in V$ has degree either 1 (ending or border point) or 3 (bifurcation). A characteristic line $c$ is a path in the graph

$$
c=\left\langle v_{1}, e_{1}, v_{2}, \ldots, e_{m-1}, v_{m}\right\rangle
$$

which connects two vertices $v_{1}, v_{m}$ of degree 1 by edges and bifurcations (vertices of degree 3 ).

Let $C$ be a set of all such paths with the additional condition that, in order to respect the natural flow, bifurcations are traversed only by following the larger angles (Fig. 2).

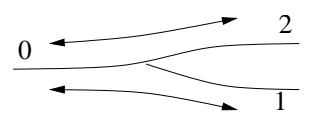

Fig. 2. Traversation of a bifurcation

\subsection{Characteristic lines and classes}

Each edge in the graph $e \in E$ is a broken line and is defined, along with the vertex points $p\left(v_{i}\right)=p_{i}=\left(X_{i}, Y_{i}\right), i \in[1, n(e)]$, by a set of $n(e)-1$ intermediate points, thus $e=\left\langle p_{1}=\left(x_{1}, y_{1}\right), p_{2}, \ldots, p_{n(e)}\right\rangle$. The metric features used for classification are the total sum of orientation changes (toc):

$$
\Phi_{t}(c)=|\Phi(c, n(e))|
$$

and the maximal orientation change (moc):

$$
\Phi_{m}(c)=\max _{k_{1}, k_{2} \in[0, n(e)]}\left|\Phi\left(c, k_{1}\right)-\Phi\left(c, k_{2}\right)\right|,
$$

where $\operatorname{Phi}(c, k)$ denotes the sum of arc changes in $E$ :

$$
\Phi(c, k)=\sum_{i=1}^{k} \angle\left(p_{i}-p_{i-1}, p_{i+1}-p_{i}\right)
$$

The changes in orientation of a connected line around the centre of the finger appear to be a simple, class - distinctive value. For the main six Henry classes they vary around the following typical values ${ }^{5}$ :

\begin{tabular}{l||c|c|c|c|c} 
class & arch & tended arch & left and right loop & whorl & twin loop \\
\hline \hline toc & 0 & 0 & $\pi$ & $2 \pi$ & $\gg 2 \pi$ \\
\hline moc & $\frac{\pi}{3}$ & $\pi$ & $\pi$ & $2 \pi$ & $\gg 2 \pi$
\end{tabular}

\footnotetext{
${ }^{5}$ The distinction between left and right loops is done by considering the orientation/position of the start point
} 


\subsection{Statistical analysis}

As mentioned in the previous section, the total orientation change $\Phi_{t}($.$) and$ the maximal orientation change $\Phi_{m}($.$) of a characteristic line are a good dis-$ tinctive measure for classifying fingerprints.

In order to find representants for each class, in a training set of fingerprints whose classes are known, a density of those two features for all characteristic lines of all fingerprints in one class was estimated. Fig. 3 illustrates computed densities of the maximal orientation change.

Consequently a fingerprint is classified according to the maximum likelihood of its feature vector against estimated densities from the training set.

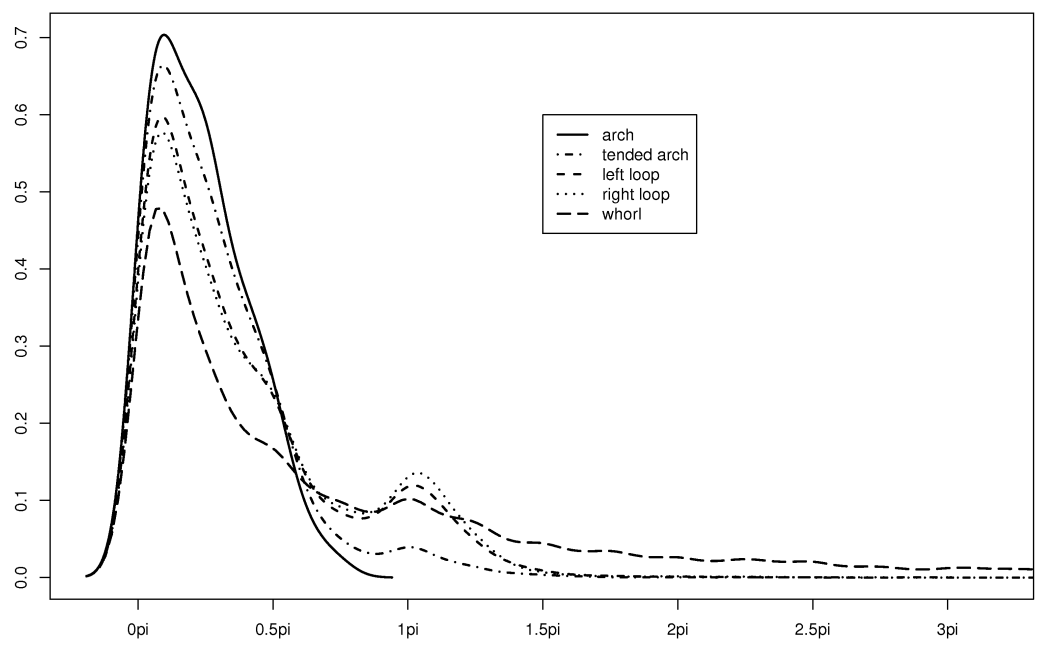

Fig. 3. Representants for all classes.

On the test database (230 images from the dataset db02 in the FVC2002 database[1]) the classifier achieves an accuracy of $88.2 \%$; the confusion matrix is reported in Table 1 . The results compare well with state of the art publications and require very few computations.

\section{Conclusions}

We presented a new approach to fingerprint classification, which uses ridge data extracted by means of entropy sensitive tracing [12]. The approach goes in the direction of characteristic lines and is expected to have an appealing ballance between the effective amount of information and operations used for class distinction and the accuracy of the classification. The papers covers preliminary tests and statistics which encourage this hypothesis. 


\begin{tabular}{c||c|c|c|c|c|c|c} 
& A & TA & LL & RL & W & DL \\
\hline \hline A & 36 & 3 & 1 & 0 & 0 & 0 \\
\hline TA & 2 & 29 & 0 & 1 & 0 & 0 \\
\hline LL & 1 & 1 & 37 & 0 & 0 & 1 \\
\hline RL & 1 & 2 & 0 & 38 & 2 & 1 \\
\hline W & 0 & 0 & 1 & 0 & 29 & 3 \\
\hline DL & 0 & 0 & 2 & 1 & 4 & 34
\end{tabular}

Table 1. Confusion matrix.

\section{References}

1. Davide Maltoni, Dario Maio, Anil K. Jain, Salil Prabhakar. Handbook of fingerprint recognition. Springer Verlag 2003.

2. Ballan, M., Sakarya, F. and Evans, B.: A Fingerprint classification technique using directional images. in 31st Asilomar conference on Signals, Systems and Computers, 1, 101-104.

3. Mardia, K., Baczkowski, A., Feng, X. and Hainsworth. T.: Statistical Methods for Automatic Interpretation of Digitally Scanned Fingerprints. Pattern Recognition Letters, 18, 1197-1203 (1997).

4. Kawagoe, M. and Tojo, A.: Fingerprint pattern classification. Pattern Recognition, 17, 295-303 (1984).

5. Senior, A.: A hidden Markov model fingerprint classifier. In proc. of the 31st Asilomar conference on Signals, Systems and Computers, 306-310 (1997)

6. Cappelli, R., Maio, D., Maltoni, D.: Fingerprint classification based on Multi-space KL. In: Proc. of AutoID'99 (Workshop on Automatic Identification Advances Technologies), 117-120 (1999)

7. Cappelli, R., Maio, D., Maltoni, D.: Multi-space KL for pattern representation and classification. IEEE Transactions on Pattern Analysis and Machine Intelligence. (23), 977-996 (2001)

8. Hong, L., Wan, Y., Jain, A.: Fingerprint image enhancement: algorithm and performance evaluation. IEEE Trans. on pattern analysis and machine intelligence. 20, 777-789 (1998)

9. Fritz, A.P., Green R.J.: Fingerprint classification using a hexagonal fast Fourier transform. Pattern recognition, 29, 1587-1597 (1996)

10. Ehrhardt, J.C.: Hexagonal Fast Fourier Transform with rectangular output. IEEE Trans. on Signal Proceeding, 41, 1469-1472 (1993)

11. Maio, D., Maltoni, D.: A structural approach to fingerprint classification. In: Proc. of the International Conference on Pattern Recognition, 578-592 (1996)

12. Mihăilescu, P., Mieloch, K., Munk, A.: Entropy sensitive fingerprint minutiae extraction, submitted. 